\title{
A Novel 3-(8-Chloro-6-(trifluoromethyl)imidazo[1,2-a]pyridine-2-yl)phenyl Acetate Skeleton and Pharmacophore Model as Glucagon-like Peptide 1 Receptor Agonists
}

\author{
Young-Dae Gong, ${ }^{*}$ Hyae Gyeong Cheon, ${ }^{\dagger}$ Taeho Lee, ${ }^{\ddagger}$ and Nam Sook Kang ${ }^{\ddagger} *$ \\ Innovative Drug Library Research Center, Department of Chemistry, Dongguk University-Seoul, Seoul 100-715, Korea \\ ${ }^{*}$ E-mail: ydgong@dongguk.edu \\ ${ }^{\dagger}$ Department of Pharmacology, Medical School, Gachon University of Medicine and Science, Incheon 406-799, Korea \\ ${ }^{\ddagger}$ Bio-Organic Science Division, Korea Research Institute of Chemical Technology, P.O. Box 107, Singseongno, \\ Yuseong-gu, Daejeon 305-600, Korea. *E-mail: nskang@krict.re.kr \\ Received September 14, 2010, Accepted October 25, 2010
}

\begin{abstract}
We screened 10,000 heterocyclic small molecules and identified a novel hit core skeleton of 3-(8-chloro-6-(trifluoromethyl)imidazo[1,2-a]pyridine-2-yl)phenyl acetate derivatives. It has been selected as a potential glucagon-like peptide 1 receptor (GLP-1R) activator and demonstrated its effects in increasing GLP-1 secretion, and thereby increasing the glucose responsiveness in both in vitro and pharmacology analyses. Further studies are currently underway to optimize the potency and selectivity of 3-(8-chloro-6-(trifluoromethyl)imidazo[1,2-a]pyridine-2-yl)phenyl acetate derivatives (hit compounds $\mathbf{2}$ and $\mathbf{8}$ ), and address their in vivo efficacy and therapeutic potential. These molecules may serve as useful evidence showing that compounds with a 3-(8-chloro-6-(trifluoromethyl)imidazo[1,2- $a$ ]pyridine-2-yl)phenyl acetate moiety are selective GLP-1R agonists, and have potential as anti-diabetic treatment agents.
\end{abstract}

Key Words: Imidazo[1,2-a]pyridine-2-yl-phenyl acetate, Glucagon-like peptide 1 receptor (GLP-1R), Druglike core skeleton, Pharmacophore

\section{Introduction}

The recent discovery of the nonpeptidic glucagon-like peptide 1 receptor (GLP-1R) agonists has attracted strong research attention as it has the potential to offer a magic bullet treatment for diabetes. ${ }^{1,2}$ Exploitation of the incretin GLP-1R has afforded effective pharmacological agents for the treatment of diabetes. Glucagon-like peptide 1 (GLP-1) has a number of propitious effects on glucose control. It directly stimulates insulin release from the pancreatic $\beta$ cell and suppresses the release of glucagon from the $\alpha$ cell. Gastric emptying is slowed by GLP-1 administration, thus slowing digestion and absorption, and moderating blood glucose excursions. Acute central or peripheral GLP-1 administration suppresses the appetite in animals and humans, and chronically reduces body weight. Particularly, GLP-1 has been reported to increase $\beta$ cell mass in rodents, reduce cell apoptosis and increase the glucose responsiveness of rodent and human islets in vitro, and stimulate the differentiation of rodent and human islet precursor cells into $\beta$ cells. ${ }^{3-5}$

The importance of GLP-1R in the mediation of normal and pathological processes has motivated considerable efforts to identify GLP-1R agonists. Although a wealth of activator compounds are available, synthesizing small-molecule GLP-1R agonists with a novel core skeleton fitting individual GLP-1R have proven to be a challenge. Many reports exist describing the development of anti-diabetic agents targeting GLP-1R, but only some anti-diabetic agents, incorporating heterocyclic rings and several aromatic core skeletons, have been identified as GLP-1R agonists. ${ }^{6}$ We, therefore, undertook an investigation aimed at developing novel and simple drug-like heterocyclic derivatives as GLP-1R agonists. By using the bioisostereo con- cept based on benzene ring-fused bicyclic GLP-1R agonists, we have designed scaffolds that combine the pyridine moiety found in the imidazo[1,2-a]pyridine-2-yl-phenylacetate ring system.

Herein, we report initial evidence showing that compounds with a 3-(8-chloro-6-(trifluoromethyl)imidazo[1,2-a]pyridine2-yl)phenyl acetate moiety are selective GLP-1R agonists, and have potential as anti-diabetic treatment agents. The aim of this study was to investigate the biochemical properties of these GLP-1R agonists and identify their new core skeleton. To identify the small-molecule agonist of GLP-1R, we screened a diverse library against HEK293 cells stably transfected with a rat GLP-1R vector and a multiple response element/cAMP response element-driven luciferase reporter plasmid (HEK293-rGLP-1R cells). The in-house library contained 10,000 heterocyclic compounds including imidazole, benzopyrans, oxazoles, thiazoles, thiadiazoles, and pyridines. ${ }^{8-12}$ We analyzed our library using the Discovery Studio Library Analysis Toolkit ${ }^{13}$ to generate a subset of diverse ligands comprising 240 compounds. The library diversity was analyzed on the basis of the following properties: cLogP, molecular weight, number of hydrogen-bond donors, number of hydrogen-bond acceptors, number of rotatable bonds, number of aromatic rings, molecular polar surface area, N plus $\mathrm{O}$ count, and extended connectivity fingerprints (ECFP_4) fin-

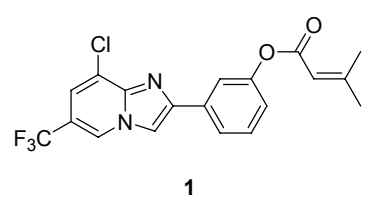

Figure 1. Structure of GLP-1R activators screened as the primary hit compound 1. 
gerprint $4{ }^{14}$ The primary screen identified a 3-(8-chloro-6-(trifluoromethyl)imidazo[1,2-a] pyridine-2-yl)phenyl-2-methyl2-butenoate, as the GLP-1R agonist as shown Figure 1, compound 1.

\section{Results and Discussion}

To clearly investigate the pharmacophoric feature of the primary hit compound shown in Figure 1, we selected three known compound of GLP-1R agonists from the Prous site (http://prous. integrity.com), by analyzing the diversity among the known compounds carried out with a maximum dissimilarity study using ECFP. After 3D structures of these compounds were generated using CONCORD, ${ }^{15}$ they were minimized using Gasteiger-Huckel charges and conjugated gradient methods. Figure 2 shows the three reference compounds ${ }^{16-18}$ selected from the Prous site and also the pharmacophore model. Common feature pharmacophores were generated using the HipHop algorithm. ${ }^{19}$ Predefined pharmacophore features were used to create the pharmacophores hypothesis model automatically. The list of features of minimum and maximum values was as follows: $\mathrm{H}$-bond acceptor (Hba) 0 and 5, $\mathrm{H}$-bond donor (Hbd) 0 and 5, and hydrophobic (Hy) 0 and 5. The compounds in the training set showed fit values ranging from 2.9 to 4.0 , compared to a range of 0.5 to 4.2 for our compounds. The pharmacophore mapping for reference 1 (see Figure 2a) having a fit value of 4.17 is shown

(a)

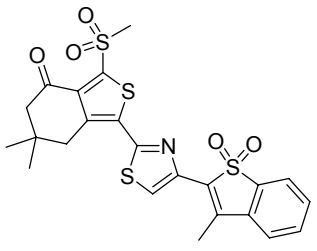

Ref. 16 : $417041(4.17)$

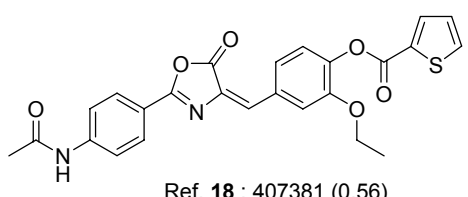

(b) Fitvalue (4.17)

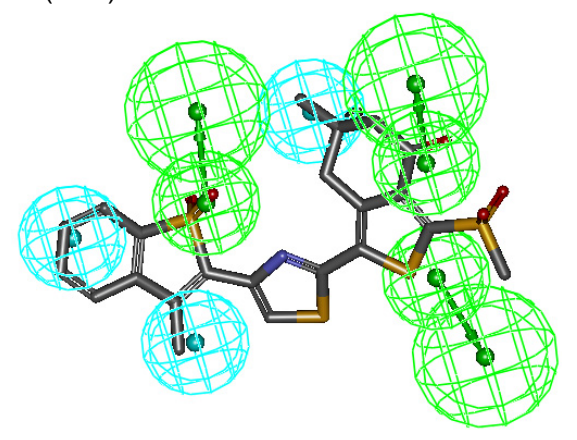

Figure 2. The reference compound of GLP-1R agonists in the training set obtained from the Prous site (http://prous.integrity.com) for the pharmacophore model: (a) the three reference compounds and (b) the corresponding pharmacophore model. The green and cyan spheres represent the hydrogen-bond acceptor and hydrophobic sites, respectively. All compounds in the training set above have a fit value of 0.5 or more. in Figure 2b. The green and cyan spheres represent the hydrogen-bond acceptor and hydrophobic sites, respectively.

We carried out pharmacophore mapping for hit compound 1 with the pharmacophore model generated by the three reference compounds, as shown in Figure 3, and found that the acetate group may be important for GLP-1R activity. Our library contained several 3-(8-chloro-6-(trifluoromethyl)imidazo[1,2- $a$ ] pyridine-2-yl)phenyl derivatives, including phenyl acetate derivatives.

Therefore, we performed secondary screening for several 3-(8-chloro-6-(trifluoromethyl) imidazo[1,2-a]pyridine-2-yl) phenyl derivatives against GLP-1R. Despite their structural similarity to the imidazo[1,2-a]pyridine-2-yl-phenyl scaffold, various functional groups substituted with the imidazo[1,2- $a$ ] pyridine-2-yl core skeleton instead of the 8-chloro and 6-trifluoromethyl positions from the library showed lower or no GLP-1R agonist activity in the secondary screening, depending on the existence of the acetate group, as shown in Figure 4.

Next, we examined the cell expression effect of the three selected more active hit compounds, 2,8 and 17, which were chosen from the first screening, on the GLP-1R agonist effect in the reporter assay determined above. Three initial hits, which had 3-(8-chloro-6-(trifluoromethyl)imidazo[1,2- $a$ ]pyridine-2yl)phenyl acetate derivatives, $\mathbf{2}, \mathbf{8}$ and $\mathbf{1 7}$, were confirmed to have target specificity toward GLP-1R and without GLP-1R expression, as shown in Figure 5, (A), (B), and (C). Compounds $\mathbf{2 ,} \mathbf{8}$ and 17 exhibited the impediment of cell proliferation in a dose-dependant manner.

In summary, we screened 10,000 heterocyclic small molecules to identify a novel hit core skeleton of 3-(8-chloro-6-(trifluoromethyl)imidazo[1,2- $a$ ]pyridine-2-yl)phenyl acetate derivatives, which was selected as a potential GLP-1R activator, and demonstrated its effects in increasing GLP-1 secretion and thereby increasing the glucose responsiveness in both in vitro and pharmacology analyses. We are presently conducting further studies to optimize the potency and selectivity of these 3(8-chloro-6-(trifluoromethyl)imidazo[1,2-a]pyridine-2-yl) phenyl acetate derivatives (compounds $\mathbf{2}$ and $\mathbf{8}$ ) and address their in vivo efficacy and therapeutic potential. These molecules may serve as useful mechanistic probes to analyze the cellular function of GLP-1R and the diabetic potential of biological mechanisms underlying compulsive chronic diabetes agents.

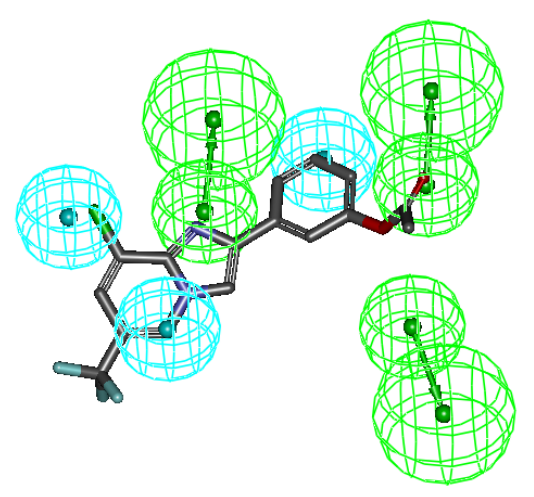

Figure 3. The pharmacophore model for hit compound $\mathbf{1}$, having the fit value of 2.99 . 
<smiles>CC(=O)Oc1cccc(-c2cn3cc(C(F)(F)F)cc(Cl)c3n2)c1</smiles><smiles>CC(C)(C)C(=O)Oc1cccc(-c2cn3cc(C(F)(F)F)cc(Cl)c3n2)c1</smiles><smiles>O=C(Oc1cccc(-c2cn3cc(C(F)(F)F)cc(Cl)c3n2)c1)C1CC1</smiles>

(2.7 folds)<smiles>O=C(Oc1cccc(-c2cn3cc(C(F)(F)F)cc(Cl)c3n2)c1)c1ccc(Cl)cc1</smiles>
(2.4 folds)<smiles>O=C(Oc1cccc(-c2cn3cc(C(F)(F)F)cc(Cl)c3n2)c1)c1ccccc1C(F)(F)F</smiles><smiles>COc1ccc(C(=O)Oc2cccc(-c3cn4cc(C(F)(F)F)cc(Cl)c4n3)c2)cc1OC</smiles><smiles>C=CC(=O)Oc1cccc(-c2cn3cc(C(F)(F)F)cc(Cl)c3n2)c1</smiles><smiles>CC(C)(C)CC(=O)Oc1cccc(-c2cn3cc(C(F)(F)F)cc(Cl)c3n2)c1</smiles>

(1.8 folds)<smiles>O=C(Oc1cccc(-c2cn3cc(C(F)(F)F)cc(Cl)c3n2)c1)C1CCCCC1</smiles><smiles>O=C(Oc1cccc(-c2cn3cc(C(F)(F)F)cc(Cl)c3n2)c1)c1ccccc1F</smiles><smiles>O=C(Oc1cccc(-c2cn3cc(C(F)(F)F)cc(Cl)c3n2)c1)c1ccc([N+](=O)[O-])cc1</smiles><smiles>CC(C)(C)c1ccc(C(=O)Oc2cccc(-c3cn4cc(C(F)(F)F)cc(Cl)c4n3)c2)cc1</smiles><smiles>CCCCCC(=O)Oc1cccc(-c2cn3cc(C(F)(F)F)cc(Cl)c3n2)c1</smiles><smiles>O=C(Oc1cccc(-c2cn3cc(C(F)(F)F)cc(Cl)c3n2)c1)c1ccccc1Cl</smiles><smiles>O=C(Oc1cccc(-c2cn3cc(C(F)(F)F)cc(Cl)c3n2)c1)c1c(F)cccc1F</smiles>

12

(1.3 folds)<smiles>Nc1ccc(C(=O)Oc2cccc(-c3cn4cc(C(F)(F)F)cc(Cl)c4n3)c2)cc1</smiles><smiles>O=C(Oc1cccc(-c2cn3cc(C(F)(F)F)cc(Cl)c3n2)c1)c1cccs1</smiles>

Figure 4. Structure of GLP-1R activators (2-18) based on 3-(8-chloro-6-(trifluoromethyl) imidazo[1,2-a]pyridine-2-yl)phenyl acetate structures identified in secondary screening toward human GLP (hGLP)-1R reporter assay in CHO-hGLP1R and CHO cells at $10 \mu \mathrm{M}$. Folds mean comparison with control.

(A)

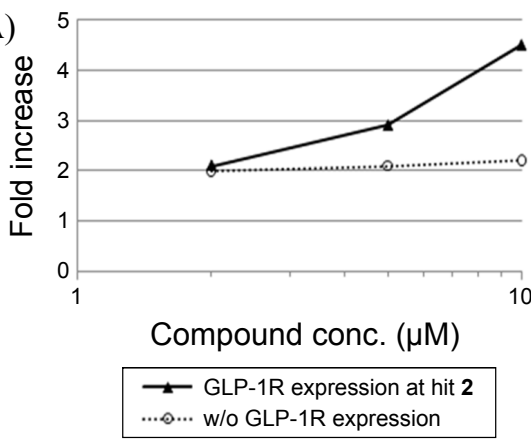

(B)

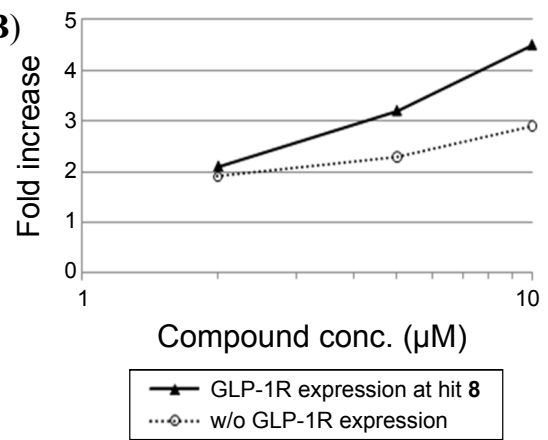

(C)

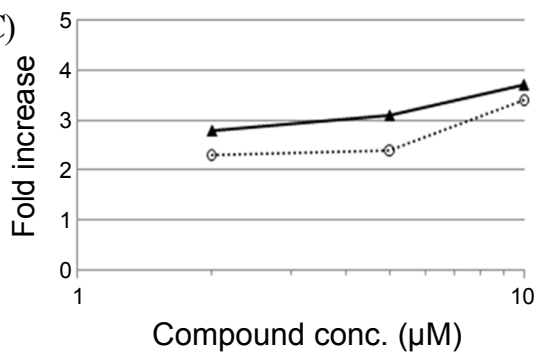

$\mp$ GLP-1R expression at hit 17 w/o GLP-1R expression

Figure 5. Three initial hits, 2(A), 8(B), and 17(C), were confirmed to have target specificity toward GLP-1R and without GLP-1R expression using luciferase assay system. Folds mean comparison with control.

\section{Experimental Procedure}

General synthesis. All chemicals were reagent grade and used as purchased. Reactions were monitored by TLC analysis using Merck silica gel 60 F-254 thin layer plates or ATR-FTIR analysis using TravelIR ${ }^{\mathrm{TM}}$ (SensIR Technology). Flash column chromatography was carried out on Merck silica gel 60 (230 -
400 mesh). ${ }^{1} \mathrm{H}$ NMR and ${ }^{13} \mathrm{C}$ NMR spectra were recorded in $\delta$ units relative to deuterated solvent as an internal reference using a Bruker $500 \mathrm{MHz}$ NMR instrument. LC-MS analysis was performed on an ESI mass spectrometer with PDA detection. The percentage LC-MS area purities of all products were determined by LC peak area analysis (XTerraMS $\mathrm{C}_{18}$ column, $4.6 \mathrm{~mm} \times$ $100 \mathrm{~mm}$; PDA detector at $200-400 \mathrm{~nm}$; gradient, 5 - 95\% 


\section{$\mathrm{CH}_{3} \mathrm{CN} / \mathrm{H}_{2} \mathrm{O}$ ).}

Representative synthetic procedure for 3-(8-chloro-6-(trifluoromethyl)imidazo[1,2-a]pyridine-2-yl)phenyl acetate (2): We prepared 3-(8-chloro-6-(trifluoromethyl)imidazo[1,2-a] ptridine-2-yl)phenol as a precursor of the final products by a previously reported method according to the following reaction scheme. $^{20}$

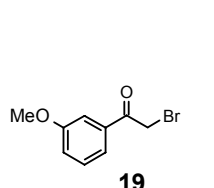

19
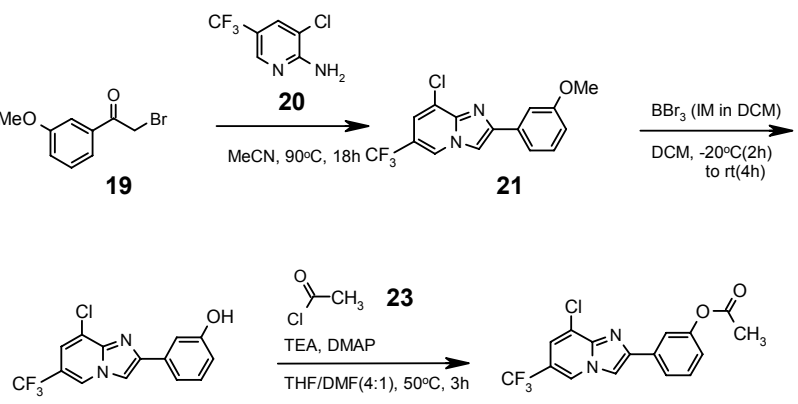

22
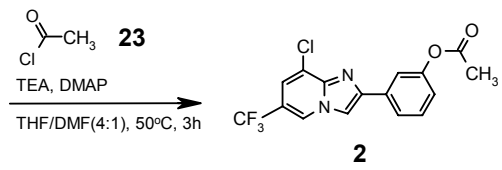

Then, to a mixture of 3-(8-chloro-6-(trifluoromethyl)imidazo [1,2-a]ptridine-2-yl)phenol $(40.0 \mathrm{mg}, 0.128 \mathrm{mM})$ in a mixture of tetrahydrofuran (THF) and $N, N$-dimethylformamide (DMF) $(4: 1,3 \mathrm{~mL})$ solution at room temperature was added acetyl chloride (12.1 mg, $0.154 \mathrm{mM}, 1.2$ equiv.) and triethylamine (19.4 $\mathrm{mg}, 0.192 \mathrm{mM}, 1.5$ equiv.) with $4-N, N$-dimethylamino pyridine (DMAP, cat. 0.1 equiv.). The resulting mixture was heated at $50{ }^{\circ} \mathrm{C}$ for $3 \mathrm{~h}$. Evaporation of the solvent gave a residue which was purified by column chromatograph [ethylacetate/hexane $1 / 4(\mathrm{v} / \mathrm{v})$ as eluent] to yield the desired 3-(8-chloro-6-(trimethyl)imidazo[1,2-a]pyridine-2-yl)phenyl acetate product, 2 (32.7 mg, 72\%). ${ }^{1} \mathrm{H}$ NMR $\left(500 \mathrm{MHz}, \mathrm{CDCl}_{3}\right) \delta 8.41-8.40(\mathrm{~m}$, 1H), 7.93 (s, 1H), 7.77-7.74 (m, 2H), 7.43-7.40 (m, 2H), 7.09 $(\mathrm{m}, 1 \mathrm{H}), 2.33(\mathrm{~s}, 3 \mathrm{H}) ;{ }^{13} \mathrm{C}$ NMR $\left(125 \mathrm{MHz}, \mathrm{CDCl}_{3}\right) \delta 169.6$, 151.3, 147.4, 142.9, 134.2, 130.0, 124.6, 123.9, 123.4, 122.2, $121.9,119.8,117.2,116.9,111.3,21.3 ;$ LC/MS (ESI) $\mathrm{m} / z 355$ $\left([\mathrm{M}+\mathrm{H}]^{+}\right)$.

3-(8-Chloro-6-(trifluoromethyl)imidazo[1,2-a]pyridine-2yl)phenyl acrylate (3): ${ }^{1} \mathrm{H}$ NMR $\left(500 \mathrm{MHz}, \mathrm{CDCl}_{3}\right) \delta 8.42-8.41$ $(\mathrm{m}, 1 \mathrm{H}), 7.95(\mathrm{~s}, 1 \mathrm{H}), 7.80-7.78(\mathrm{~m}, 2 \mathrm{H}), 7.44-7.40(\mathrm{~m}, 2 \mathrm{H}), 7.13$ $(\mathrm{m}, 1 \mathrm{H}), 6.66-6.62(\mathrm{~m}, 1 \mathrm{H}), 6.38-6.33(\mathrm{~m}, 1 \mathrm{H}), 6.06-6.04(\mathrm{~m}$, $1 \mathrm{H})$.

3-(8-Chloro-6-(trifluoromethyl)imidazo[1,2-a]pyridine-2yl)phenyl pivalate (4): ${ }^{1} \mathrm{H} N \mathrm{NM}\left(500 \mathrm{MHz}, \mathrm{CDCl}_{3}\right) \delta 8.38$ (s, $1 \mathrm{H}), 7.86(\mathrm{~m}, 1 \mathrm{H}), 7.70(\mathrm{~s}, 1 \mathrm{H}), 7.64-7.62(\mathrm{~m}, 1 \mathrm{H}), 7.37-7.31$ $(\mathrm{m}, 2 \mathrm{H}), 7.01-7.00(\mathrm{~m}, 1 \mathrm{H}), 1.40(\mathrm{~s}, 9 \mathrm{H})$.

3-(8-Chloro-6-(trifluoromethyl)imidazo[1,2-a]pyridine-2yl)phenyl-3,3-dimethyl butanoate (5): ${ }^{1} \mathrm{H}$ NMR $(500 \mathrm{MHz}$, $\left.\mathrm{CDCl}_{3}\right) \delta 8.37(\mathrm{~m}, 1 \mathrm{H}), 7.87-7.86(\mathrm{~m}, 1 \mathrm{H}), 7.68-7.67(\mathrm{~m}, 2 \mathrm{H})$, 7.37-7.34 (m, 2H), 7.04-7.02 (m, 1H), 2.48 (s, 2H), $1.17(\mathrm{~s}, 9 \mathrm{H})$.

3-(8-Chloro-6-(trifluoromethyl)imidazo[1,2-a]pyridine-2yl)phenylhexanoate (6): ${ }^{1} \mathrm{H} \mathrm{NMR}\left(500 \mathrm{MHz}, \mathrm{CDCl}_{3}\right) \delta 8.37$ (s, $1 \mathrm{H}), 7.85(\mathrm{~m}, 1 \mathrm{H}), 7.70-7.65(\mathrm{~m}, 2 \mathrm{H}), 7.36-7.34(\mathrm{~m}, 2 \mathrm{H}), 7.04-$ $7.02(\mathrm{~m}, 1 \mathrm{H}), 2.60(\mathrm{t}, 2 \mathrm{H}, J=7.8 \mathrm{~Hz}), 1.80-1.74(\mathrm{~m}, 2 \mathrm{H}), 1.51-$ $1.44(\mathrm{~m}, 2 \mathrm{H}), 1.25-0.98(\mathrm{~m}, 3 \mathrm{H})$.

3-(8-Chloro-6-(trifluoromethyl)imidazo[1,2-a]pyridine-2yl)phenylcyclopropane carboxylate (7): ${ }^{1} \mathrm{H}$ NMR $(500 \mathrm{MHz}$, $\left.\mathrm{CDCl}_{3}\right) \delta 8.37(\mathrm{~s}, 1 \mathrm{H}), 7.85(\mathrm{~s}, 1 \mathrm{H}), 7.72-7.71(\mathrm{~m}, 1 \mathrm{H}), 7.67-$ $7.65(\mathrm{~m}, 1 \mathrm{H}), 7.37-7.33(\mathrm{~m}, 2 \mathrm{H}), 7.26-7.06(\mathrm{~m}, 1 \mathrm{H}), 1.89-1.86$ $(\mathrm{m}, 1 \mathrm{H}), 1.22-1.19(\mathrm{~m}, 2 \mathrm{H}), 1.07-1.03(\mathrm{~m}, 2 \mathrm{H})$.

3-(8-Chloro-6-(trifluoromethyl)imidazo[1,2- $a$ ]pyridine-2yl)phenylcyclohexane carboxylate (8): ${ }^{1} \mathrm{H}$ NMR $(500 \mathrm{MHz}$, $\left.\mathrm{CDCl}_{3}\right) \delta 8.37(\mathrm{~s}, 1 \mathrm{H}), 7.84(\mathrm{~s}, 1 \mathrm{H}), 7.68(\mathrm{~m}, 1 \mathrm{H}), 7.63-7.62(\mathrm{~m}$, $1 \mathrm{H}), 7.36-7.30(\mathrm{~m}, 2 \mathrm{H}), 7.01-6.99(\mathrm{~m}, 1 \mathrm{H}), 2.59-2.58(\mathrm{~m}, 1 \mathrm{H})$, 2.12-2.09 (m, 2H), 1.86-1.83 (m, 2H), 1.63-1.61 (m, 3H), 1.401.32 (m, 3H); LC/MS (ESI) $\mathrm{m} / z 422\left([\mathrm{M}]^{+}\right)$.

3-(8-Chloro-6-(trifluoromethyl)imidazo[1,2-a]pyridine-2yl)phenyl-2-chlorobenzoate (9): ${ }^{1} \mathrm{H}$ NMR $\left(500 \mathrm{MHz}, \mathrm{CDCl}_{3}\right)$ $\delta 8.38(\mathrm{~s}, 1 \mathrm{H}), 8.15-8.13(\mathrm{~m}, 2 \mathrm{H}), 7.91(\mathrm{~s}, 1 \mathrm{H}), 7.85(\mathrm{~m}, 1 \mathrm{H})$, $7.74(\mathrm{~m}, 1 \mathrm{H}), 7.50-7.48(\mathrm{~m}, 2 \mathrm{H}), 7.42-7.41(\mathrm{~m}, 1 \mathrm{H}), 7.37(\mathrm{~m}$, $1 \mathrm{H}), 7.19(\mathrm{~m}, 1 \mathrm{H})$.

3-(8-Chloro-6-(trifluoromethyl)imidazo[1,2-a]pyridine-2yl)phenyl-4-chlorobenzoate (10): ${ }^{1} \mathrm{H} \mathrm{NMR}\left(500 \mathrm{MHz}, \mathrm{CDCl}_{3}\right)$ $\delta 8.39(\mathrm{~s}, 1 \mathrm{H}), 8.15-8.13(\mathrm{~m}, 2 \mathrm{H}), 7.91(\mathrm{~s}, 1 \mathrm{H}), 7.86-7.85(\mathrm{~m}$, 1H), $7.75(\mathrm{~d}, 1 \mathrm{H}, J=7.8 \mathrm{~Hz}), 7.50-7.48(\mathrm{~m}, 2 \mathrm{H}), 7.43-7.41(\mathrm{~m}$, $1 \mathrm{H}), 7.37(\mathrm{~m}, 1 \mathrm{H}), 7.19(\mathrm{~m}, 1 \mathrm{H})$.

3-(8-Chloro-6-(trifluoromethyl)imidazo[1,2-a]pyridine-2yl)phenyl-2-fluorobenzoate (11): ${ }^{1} \mathrm{H} \mathrm{NMR}\left(500 \mathrm{MHz}, \mathrm{CDCl}_{3}\right)$ $\delta 8.40(\mathrm{~s}, 1 \mathrm{H}), 8.13-8.12(\mathrm{~m}, 1 \mathrm{H}), 7.92(\mathrm{~s}, 1 \mathrm{H}), 7.86(\mathrm{~m}, 1 \mathrm{H})$, $7.77(\mathrm{~d}, 1 \mathrm{H}, J=7.8 \mathrm{~Hz}), 7.65(\mathrm{~m}, 1 \mathrm{H}), 7.44-7.41(\mathrm{~m}, 1 \mathrm{H}), 7.3$ (m, 1H), 7.29-7.20 (m, 3H).

3-(8-Chloro-6-(trifluoromethyl))imidazo[1,2-a]pyridine2-yl)phenyl-2,6-difluorobenzoate (12): ${ }^{1} \mathrm{H}$ NMR $(500 \mathrm{MHz}$, $\left.\mathrm{CDCl}_{3}\right) \delta 8.42(\mathrm{~s}, 1 \mathrm{H}), 7.97(\mathrm{~s}, 1 \mathrm{H}), 7.87-7.82(\mathrm{~m}, 2 \mathrm{H}), 7.51-7.44$ (m, 2H), $7.39(\mathrm{~m}, 1 \mathrm{H}), 7.26-7.25(\mathrm{~m}, 1 \mathrm{H}), 7.06-7.03(\mathrm{~m}, 2 \mathrm{H})$.

3-(8-Chloro-6-(trifluoromethyl)imidazo[1,2- $a]$ pyridine2-yl)phenyl-2-trifluoromethyl benzoate (13): ${ }^{1} \mathrm{H}$ NMR (500 $\left.\mathrm{MHz}, \mathrm{CDCl}_{3}\right) \delta 8.43(\mathrm{~s}, 1 \mathrm{H}), 8.00(\mathrm{~m}, 2 \mathrm{H}), 7.88-7.84(\mathrm{~m}, 3 \mathrm{H})$, 7.72-7.70 (m, 2H), 7.51-7.48 (m, 1H), 7.41 (m, 1H), $7.26(\mathrm{~m}$, $1 \mathrm{H})$.

3-(8-Chloro-6-(trifluoromethyl)imidazo[1,2-a]pyridine-2yl)phenyl-4-nitrobenzoate (14): ${ }^{1} \mathrm{H} \mathrm{NMR}\left(500 \mathrm{MHz}, \mathrm{CDCl}_{3}\right)$

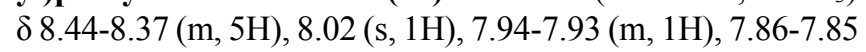
$(\mathrm{m}, 1 \mathrm{H}), 7.54-7.50(\mathrm{~m}, 1 \mathrm{H}), 7.42(\mathrm{~m}, 1 \mathrm{H}), 7.26(\mathrm{~m}, 1 \mathrm{H})$.

3-(8-Chloro-6-(trifluoromethyl)imidazo[1,2-a]pyridine-2yl)phenyl-4-cyanobenzoate (15): ${ }^{1} \mathrm{H} \mathrm{NMR}\left(500 \mathrm{MHz}, \mathrm{CDCl}_{3}\right)$ $\delta 8.44-8.37(\mathrm{~m}, 5 \mathrm{H}), 8.02(\mathrm{~s}, 1 \mathrm{H}), 7.94-7.93(\mathrm{~m}, 1 \mathrm{H}), 7.86-7.85$ $(\mathrm{m}, 1 \mathrm{H}), 7.54-7.50(\mathrm{~m}, 1 \mathrm{H}), 7.42(\mathrm{~m}, 1 \mathrm{H}), 7.26(\mathrm{~m}, 1 \mathrm{H})$.

3-(8-Chloro-6-(trifluoromethyl)imidazo[1,2-a]pyridine-2yl)phenyl-3,4-dimethoxy benzoate (16): ${ }^{1} \mathrm{H}$ NMR $(500 \mathrm{MHz}$, $\left.\mathrm{CDCl}_{3}\right) \delta 8.43(\mathrm{~s}, 1 \mathrm{H}), 7.98(\mathrm{~s}, 1 \mathrm{H}), 7.90-7.87(\mathrm{~m}, 2 \mathrm{H}), 7.83-$ $7.82(\mathrm{~m}, 1 \mathrm{H}), 7.70-7.69(\mathrm{~m}, 1 \mathrm{H}), 7.50-7.46(\mathrm{~m}, 1 \mathrm{H}), 7.41-7.40$ $(\mathrm{m}, 1 \mathrm{H}), 7.26(\mathrm{~m}, 1 \mathrm{H}), 6.97(\mathrm{~d}, 1 \mathrm{H}, J=8.5 \mathrm{~Hz}), 3.99-3.98(\mathrm{~m}$, $6 \mathrm{H})$.

3-(8-Chloro-6-(trifluoromethyl)imidazo[1,2-a]pyridine2-yl)phenyl-4-tert-butylbenzoate (17): ${ }^{1} \mathrm{H}$ NMR $(500 \mathrm{MHz}$, $\left.\mathrm{CDCl}_{3}\right) \delta 8.39(\mathrm{~s}, 1 \mathrm{H}), 8.15(\mathrm{~d}, 2 \mathrm{H}, J=8.4 \mathrm{~Hz}), 7.88(\mathrm{~m}, 1 \mathrm{H})$, $7.83(\mathrm{~m}, 1 \mathrm{H}), 7.72-7.70(\mathrm{~m}, 1 \mathrm{H}), 7.54(\mathrm{~d}, 2 \mathrm{H}, J=8.4 \mathrm{~Hz}), 7.40-$ $7.35(\mathrm{~m}, 2 \mathrm{H}), 7.17-7.16(\mathrm{~m}, 1 \mathrm{H}), 1.38(\mathrm{~s}, 9 \mathrm{H}) ;{ }^{13} \mathrm{C}$ NMR $(125$ $\left.\mathrm{MHz}, \mathrm{CDCl}_{3}\right) \delta 165.3,157.7,151.7,147.4,142.9,134.2,130.3$, $130.0,126.8,125.8,124.5,123.9,123.5,122.4,120.0,119.8$, 117.1, 116.8, 111.4, 35.4, 31.3; LC/MS (ESI) $m / z 472\left([\mathrm{M}]^{+}\right)$.

3-(8-Chloro-6-(trifluoromethyl)imidazo[1,2-a]pyridine-2yl)phenylthiophene-2-carboxylate (18): ${ }^{1} \mathrm{H}$ NMR $(500 \mathrm{MHz}$, $\left.\mathrm{CDCl}_{3}\right) \delta 8.40(\mathrm{~s}, 1 \mathrm{H}), 8.00-7.99(\mathrm{~m}, 1 \mathrm{H}), 7.93(\mathrm{~s}, 1 \mathrm{H}), 7.85(\mathrm{~m}$, $1 \mathrm{H}), 7.74(\mathrm{~m}, 1 \mathrm{H}), 7.69-7.68(\mathrm{~m}, 1 \mathrm{H}), 7.43(\mathrm{~m}, 1 \mathrm{H}), 7.38(\mathrm{~m}$, 
1H), 7.20-7.19 (m, 2H).

Preparation of the testing materials. The testing material stock plate was prepared as a concentration of 2,5 , and $10 \mathrm{mM}$ in DMSO. A 1/125 dilution of the stock solution was made by diluting $2 \mu \mathrm{L}$ in $248 \mu \mathrm{L}$ of culture media. The cell culture media was aspirated out, $175 \mu \mathrm{L}$ of fresh media was added, and $25 \mu \mathrm{L}$ of the prepared stock solution was added to the media, which gave concentrations of 2,5 , and $10 \mu \mathrm{M}$. The treated cells were incubated for 72 hours.

Activation efficacy test of compounds on CHO-hGLP1R using the reporter assay system. We followed the pioneer patent and published paper for this in vitro screening., ${ }^{2,17}$ The HEK293rGLP-1R cells were seeded onto 96-well cell culture plates with a density of 40,000 cells per well and incubated overnight. At the time of assaying, GLP-1(7-37) (Sigma, St. Louis, MO) or test compounds dissolved in DMSO were added $(1-100 \mu \mathrm{L})$. In the blocking studies, the cells were reacted with exendin (9-39) (Ana Spec, San Jose, CA) in DMSO ( $1 \mu \mathrm{L})$ for $10 \mathrm{~min}$ before the above procedure. After $6 \mathrm{~h}$ of incubation, they were lysed and quantified for luciferase activity by using the SteadyGlo luciferase assay system (Promega).

Acknowledgments. This research was supported by a grant (CBM34-B1000-01-00-01) from the Center for Biological Modulators of the 21 st Century Frontier R\&D Program from the Ministry of Education, Science and Technology, Korea and the Ministry of Knowledge Economy, Korea.

\section{References}

1. Murphy, K. G.; Bloom, S. R. Proc. Natl. Acad. Sci. USA 2007, 104, 689.
2. Chen, D.; Liao, J.; Li, N.; Zhou, C.; Liu, Q.; Wang, G.; Zhang, R.; Zhang, S.; Lin, L.; Chen, K.; Xie, X.; Nan, F.; Young, A. A.; Wang, M.-W. Proc. Natl. Acad. Sci. USA 2007, 104, 943.

3. Larsen, P. J.; Holst, J. J. Regul. Peptides 2005, 128, 97.

4. Bonner-Weir, S.; Weir, G. C. Nat. Biotechnol. 2005, 23, 857.

5. Drucker, D. J.; Nauck, M. A. Lancet. 2006, 368, 1696.

6. (a) Teng, M.; Johnson, M. D.; Thomas, C.; Kiel, D.; Lakis, J. N.; Kercher, T.; Aytes, S.; Kostrowicki, J.; Bhumralkar, D.; Truesdale, L.; May, J.; Sidelman, U.; Kodra, J. T.; Jørgensen, A. S.; Olesen, P. H.; de Jong, J. C.; Madsen, P.; Behrens, C.; Pettersson, I.; Kundsen, L. B.; Holst, J. J.; Lau, J. Bioorg. Med. Chem. Lett. 2007, 17, 5472. (b) Murphy, K. G.; Bloom, S. R. Proc. Natl. Acad. Sci. USA 2007, 104, 689.

7. Choi, H. J.; Song, B.-J.; Gong, Y.-D.; Gwak, W. J.; Soh, Y. Brit. J. Pharmacol. 2008, 154, 114.

8. Hwang, J. Y.; Choi, H.-S.; Seo, J.-S.; La, H.-J.; Yoo, S.-e.; Gong, Y. D. J. Comb. Chem. 2006, 8, 897.

9. Hwang, J. Y.; Gong, Y. D. J. Comb. Chem. 2006. 8, 297.

10. Hwang, J. Y.; Choi, H. S.; Lee, D. H.; Yoo, S.-e.; Gong, Y. D. J. Comb. Chem. 2005, 7, 136.

11. Hwang, J. Y.; Choi, H. S.; Lee, D. H.; Gong, Y. D. J. Comb. Chem. 2005, 7,816

12. Lee, I. Y.; Kim, S. Y.; Lee, J. Y.; Yu, C.-M.; Lee, D. H.; Gong, Y. D. Tetrahedron Lett. 2004, 45, 9319.

13. Software Inc., San Diego, 2005.

14. Hert, J.; Willett, P.; Wilton, D. J.; Acklin, P.; Azzaoui, K.; Jacoby, E.; Schuffenhauer, A. J. Chem. Inf. Comp. Sci. 2004, 44, 1177.

15. Concord; Tripos Inc., 1699 South Hanley Road, St. Louis, MO 63144.

16. Knudsen, S. M.; Petterson, I.; Lau, J.; Behrens, C.; Petersen, A. K.; Lau, J.; Garibay, P. W. PCT WO 2006/052873.

17. Lau, J. Drug. Data. Rep. 2000, 22, 1008

18. Nan, F.; Wang, W.; Zhou, C. Chinese Patent, WO 2005/056537.

19. Catalyst User Guide, Accelrys Software Inc., San Diego, 2005.

20. Leopoldo, M.; Lacivita, E.; Passafiume, E.; Contino, M.; Colabufo, N.; Berardi, F.; Perrone, R. J. Med. Chem. 2007, 50, 5043.

21. Wang, M. W.; Li, N.; Wang, G.; Xie, X.; Liu, Q.; Zhou, C.; Nan, F. Chinese Patent, PCT WO 2006/136101 Al. 